Théologiques

Théologiques

\title{
Présent absolu et avenir absolu
}

Nishida et Rahner (2)

\section{Jacynthe Tremblay}

Volume 17, numéro 2, 2009

Querelles d'images?

URI : https://id.erudit.org/iderudit/044071ar

DOI : https://doi.org/10.7202/044071ar

Aller au sommaire du numéro

\section{Éditeur(s)}

Faculté de théologie et de sciences des religions, Université de Montréal

\section{ISSN}

1188-7109 (imprimé)

1492-1413 (numérique)

Découvrir la revue

\section{Citer cet article}

Tremblay, J. (2009). Présent absolu et avenir absolu : Nishida et Rahner (2). Théologiques, 17(2), 245-263. https://doi.org/10.7202/044071ar
Résumé de l'article

De nombreux points de comparaison sont possibles entre la philosophie de Nishida Kitarō (1870-1945) et la philosophie de la religion de Karl Rahner (1904-1984), notamment concernant la temporalité, thème à propos duquel ces deux auteurs réfléchirent radicalement. En réexaminant la notion rahnérienne d'avenir absolu à la lumière de la notion nishidienne de présent absolu, il est possible d'apporter une solution au problème suivant : rejoint-on Dieu (Rahner) ou l'absolu (Nishida) dans un avenir situé au-delà de la temporalité ou bien dans l'instant présent, compris comme le lieu même du jaillissement de la temporalité ? Dans la mesure où elle s'appuie sur une philosophie du néant absolu, la pensée de Nishida permet de dégager la philosophie de la religion de Rahner des cloisons de l'onto-théo-téléologie qui l'enserrent et, la plaçant ainsi sur un horizon plus vaste, la rend apte à exprimer, d'une manière qu'on espère nouvelle, les nombreuses potentialités qu'elle recèle encore. En retour, une lecture de la temporalité nishidienne à travers le regard de Rahner permet de l'approfondir et de faire ressortir des éléments inédits. 
Théologiques 17, no 2 (2009) p. 245-263

\section{Présent absolu et avenir absolu} Nishida et Rahner $\left(2^{1}\right)$

Jacynthe TREMBLAY"

\section{Dieu comme avenir absolu}

Là où Rahner établit étroitement les liens entre son anthropologie transcendantale et la théologie proprement dite concernant le thème de l'avenir est lorsqu'il affirme que la question de l'avenir absolu de l'humain implique la question de Dieu lui-même. De telle façon que «se mettre dans une disposition d'accueil vis-à-vis de l'éventualité d'un tel avenir absolu, c'est faire du même coup l'expérience de ce que les chrétiens mettent sous le mot de Dieu " (Rahner 1966e, 147). L'avenir absolu est la réalité même de Dieu. Peu importe que l'on saisisse ou non par la réflexion que croire en un avenir absolu et croire en Dieu est une seule et même chose. La tâche de l'anthropologie transcendantale était de mettre à jour cette disposition d'accueil face à l'éventualité de l'avenir absolu. La théologie intervient lorsqu'on nomme «Dieu » cet avenir absolu. Il s'agira donc dans cet article d'examiner la nature d'une rencontre qui d'un côté part de l'humain tout entier tendu vers l'avenir absolu, et qui d'un autre côté advient à partir de l'avenir absolu qui vient vers l'humain depuis l'extrémité du futur.

Selon Rahner, l'avenir qui attend l'être humain est un avenir absolu. Rahner résume sa pensée sur la question en précisant que «le passé, [l'humain] l'interprète à la lumière de l'avenir, au fur et à mesure que celui-ci se

1. La première partie de cet article est parue dans Théologiques $17 / 1$ (2009), p. 203-228.

* Après de nombreuses années de recherche et d'enseignement au Japon et en Chine, Jacynthe Tremblay est actuellement chargée de cours à l'Université de Montréal. Ses recherches portent sur la philosophie de Nishida Kitarō (1870-1945) et sur la philosophie japonaise du $\mathrm{XX}^{\mathrm{e}}$ siècle. Parmi ses publications, on compte les titres suivants: (1992) Finitude et devenir. Fondements philosophiques du concept de révélation chez Karl Rahner, Montréal, Fides; (2000) Nishida Kitarō. Le Jeu de l'individuel et de l'universel, Paris, CNRS Éditions; (2007) Introduction à la philosophie de Nishida, Paris, L'Harmattan. 
dévoile. Le présent, il ne le comprend et ne l'apprécie qu'en refusant de s'y enfermer, qu'en étant tendu vers l'approche de l'avenir absolu» (Rahner 1966e, 149).

La réalité du devenir absolu que proclame le christianisme n'est pas un écoulement dans le vide. Il débouche sur l'avenir absolu, dans la mouvance duquel il se développe. Cette conception du devenir exclut le panthéisme car il ne peut être confondu avec son terme et son achèvement. Mais même si la réalité infinie de l'avenir est indépendante du devenir, elle n'en est pas moins « un élément interne essentiel, la loi qui le régit, la réalité qui en sous-tend le développement» (Rahner 1966e, 150). Cette conception préserve ce qu'il y a de juste dans le panthéisme, tout en étant au-delà d'un théisme simpliste et d'une conception extrinsèque des rapports entre Dieu et le monde.

Rahner définit donc l'être humain comme "un être capable d'atteindre l'avenir absolu » (Rahner 1966e, 150). Il s'agit d'un état qui apparaîtra lorsqu'aura pris fin la marche ascendante de l'humanité et la succession des étapes - chacune relative et en ordre de perfection croissante - de son développement. Ainsi, le christianisme est une religion du devenir, de l'histoire, de l'autotranscendance et de l'avenir absolu. Chaque situation impose à l'humain une tâche qui devra être accomplie (thème déjà abordé avec la question des mystiques terrestres de l'avenir); le sens de ce qui est lui est fourni par ce qui n'est pas encore. Le fondement suprême des élans humains est la plénitude absolue de Dieu. Leur terme ultime est Dieu qui se communique lui-même. "Il s'ensuit, conclut Rahner, que la véritable intelligence de l'être et de la nature des choses doit nécessairement prendre son point de départ dans le futur, dans la lumière qu'il jette sur les commencements» (Rahner 1966e, 1512).

Cela posé, et pour bien marquer la différence entre l'ordre humain et le domaine divin, Rahner reprend cette même question de l'avenir absolu par le biais de sa distinction entre le «catégorial» et le «transcendantal». L'avenir en fonction duquel s'établit le "projet» humain est-il un avenir catégorial composé d'éléments singuliers et limité spatiotemporellement? Ou bien est-il un avenir infini au-delà duquel rien ne peut plus être conçu, c'est-à-dire "un état dans lequel le champ infini des possibilités d'avenir devient une réalité présente? " (Rahner 1966e, 152).

2. À propos des rapports entre le présent et le futur, voir aussi Rahner ( «Pour une théologie de l'enfance », 428-439; L'Homme au miroir de l'année chrétienne, 1966, 11-21). 
Dans d'autres contextes, Rahner parle du «catégorial» dans le même sens que du "thématique ", c'est-à-dire du domaine des objets de connaissance et d'action. Comme la philosophie scolastique avant lui, il s'inspire en cela d'Aristote, chez qui le catégorial correspond à des objets de pensée répartis en catégories (substance, quantité, qualité, etc.). Au catégorial et au thématique, Rahner oppose le transcendantal ou le non-thématique qui forme la toile de fond ou la condition a priori sans laquelle ne serait possible la saisie d'aucun objet catégorial. Situé au-delà (trans-cendere) des catégories des objets, le transcendantal accompagne tous les objets de pensée, même les plus généraux que sont le vrai, le bien, le bon et, surtout, l'être (car tout ce qui entre dans l'esprit est quelque chose).

Dans la philosophie moderne, le transcendantal a été compris comme la condition a priori de la vie de l'esprit rendant possible les objets de pensée, de vouloir ou d'amour. Quant au catégorial, il a été relégué au domaine des objets de pensée proprement dits. À ce niveau, la perspective de Rahner rejoint celle de la philosophie moderne, à la différence que le transcendantal est dans sa perspective un horizon indéfinissable et inépuisable, quoique réel au suprême degré, et sans lequel la pensée, le vouloir et l'amour seraient inconcevables. Dans le mouvement inverse, l'expérience de cette réalité suprême se retraduit nécessairement en éléments catégoriaux tels que les idées, les raisonnements, les sentiments et les décisions qui, néanmoins, demeurent constamment en deçà de cette expérience. Le transcendantal, en effet, ne peut s'identifier avec aucun acte de pensée, de vouloir ou d'amour. Il n'est pas non plus la somme de ces objets, réels ou possibles. Ces actes laissent l'humain insatisfait puisqu'ils ne se suffisent pas à eux-mêmes; ils doivent être resitués dans le mystère absolu (Dieu) qui les enveloppe.

Pour le christianisme, le véritable avenir de l'humain est l'avenir absolu, possibilité réelle d'un destin futur dont l'acceptation constitue la tâche suprême. Alors que l'avenir fabriqué par l'humain est situé spatiotemporellement et emprunte des éléments au domaine catégorial, l'avenir absolu est "quelque chose qui, bien qu'on ne puisse s'en faire une représentation, enveloppe nécessairement tout cela sans être, lui, enveloppé par rien, limité par rien» (Rahner 1966e, 153). Cette réalité infinie qui englobe tout, tel est l'avenir absolu.

Il est déjà possible ici de commencer à établir des liens entre l'avenir absolu qui enveloppe et englobe tout, sans être enveloppé par rien, et le présent absolu tel qu'il est conçu par Nishida Kitarō (1870-1945). À titre de néant absolu, le présent absolu englobe toute chose, sans être lui-même 
englobé par rien. L'usage fait par Rahner dans ce contexte de la notion d'englobement est toutefois différent de celui de Nishida. Là où le premier mentionne que l'avenir absolu n'est limité par rien, le second parle d'un cercle illimité. Sur ce point, l'avenir absolu et le présent absolu sont structurellement en affinité. Malgré cela, il ne faudrait pas aller jusqu'à dire, avec pour intention expresse d'établir sur ce sujet entre l'avenir absolu rahnérien et le présent absolu nishidien des liens qui ne s'y trouvent pas, que le présent absolu est, comme néant, plus englobant que l'avenir absolu que Rahner identifie à Dieu. Encore une fois, Dieu et le néant ne peuvent être comparés car l'un est un étant, et l'autre pas. Mouvement d'autonégation qui vaut à tout élément de l'univers d'entrer en relation avec tout autre, le néant absolu, il ne faut pas l'oublier, affecte Dieu lui-même - même s'il n'est pas du même ordre, ontologique, que lui — et, à un niveau suréminent, son autocommunication (la révélation) et son devenir-humain (l'incarnation). Autrement dit, Dieu lui-même est affecté par le néant absolu en ce sens qu'il se nie lui-même afin de se communiquer à l'être humain, de devenir lui-même humain et d'entrer ainsi en relation avec l'humanité.

Malgré le caractère négativiste de la théologie de Rahner, il est possible d'y retrouver aussi en un certain sens ces notions nishidiennes d'autonégation et de relationalité. La différence, encore une fois, en est une de conceptions du monde, téléologique dans le cas de Rahner; qui est d'ordre topologique dans le cas de Nishida. Sur la base de la notion de lieu, on est autorisé à adresser à la problématique de Rahner les questions suivantes: comment l'avenir absolu, "réalité infinie qui englobe tout », peut-il se situer au-delà du temps et de l'histoire? Comment peut-il englober ces derniers s'il doit encore se situer à leur terme? Le problème en est surtout un de représentation, c'est-à-dire la difficulté qu'il y a à se représenter une réalité englobante qui ne pourrait être approchée qu'au terme d'un temps rectilinéaire.

Rahner semble avoir prévenu la difficulté car il ajoute: "Cet avenir absolu devient lui-même une réalité dans l'homme, car celui-ci le reçoit en partage et le possède déjà » (Rahner 1966e, 153). Il s'avère donc que les notions de devenir-humain et d'autocommunication de Dieu permettront dans une certaine mesure de solutionner les apories suscitées par les conceptions rectilinéaire du temps et eschatologique de l'histoire (voir la section suivante).

Rahner poursuit en parlant de la disposition d'accueil vis-à-vis de la réalité totale et englobante qu'est l'avenir absolu, le mystère absolu qui ne saurait devenir l'objet d'une appellation catégoriale. Par l'accueil que lui réserve l'humain, l'avenir absolu peut s'intégrer dans l'histoire de cet 
humain et devenir un événement de son existence. On constate ainsi qu'après avoir situé d'abord l'avenir absolu au terme de la temporalité et de l'histoire humaines, Rahner le réinsère dans l'histoire et l'expérience. En effet, on est autorisé à considérer que Dieu comme avenir absolu est "le terme et le moteur du dynamisme qui pousse le monde et l'homme vers l'avenir; il est le levier même, et non le terme simplement possible et encore à venir, de l'espérance qui suscite les efforts humains» (Rahner 1966e, 155). Dans cette perspective, l'avenir absolu apparaît comme la plénitude absolue du réel. Il n'est pas un objet de connaissance parmi d'autres mais ce qui donne sa consistance aux plans d'avenir humains, le mystère rebelle à toute expression de nature catégoriale.

Cette problématique rahnérienne de l'avenir absolu lui permet de dépasser toute vision utopique de l'avenir fondée sur une idéologie. Par idéologie, il entend la prétention qui confond un avenir constitué d'éléments catégoriaux avec l'avenir absolu. Ou encore le fait de soustraire à la volonté humaine son autonomie pour lui imposer en lieu et place une motivation étrangère. La notion rahnérienne d'avenir absolu se révèle d'autant plus intéressante qu'elle permet de déconstruire non seulement toute idéologie, mais aussi toute conceptualisation figée concernant Dieu et la religion.

Il apparaît au terme de ces considérations que deux conceptions de la temporalité persistent dans la théologie de Rahner. L'une se caractérise par sa négativité, tandis que l'autre présente des éléments positifs qui sont appelés par son anthropologie philosophique et par sa mise en lumière des conditions de possibilité de l'advenir de Dieu en ce monde et dans la finitude humaine. Ce sont ces deux perspectives, négative et positive, de même que leur intrication, qu'il s'agit désormais d'examiner plus en profondeur, du point de vue de la venue concrète de Dieu dans l'histoire et la temporalité humaines. Il apparaîtra au terme des prochaines analyses que la prise en compte de ce thème obligea Rahner à octroyer sa pleine positivité à l'être humain et à la temporalité, sans quoi l'autocommunication de Dieu et son devenir-humain auraient risqué d'être compris comme des éléments extrinsèques surajoutés à une nature et un devenir humains marqués irrémédiablement par la caducité, et par conséquent sans lieu possible d'insertion.

\section{Le devenir-humain de Dieu. Une conception positive de la temporalité}

En raison d'une conception au point de départ négative du créé et des affaires humaines, et par extension de la temporalité, Rahner se crut obligé se situer l'éternité au-delà du temps, c'est-à-dire à son terme. Il se conforma 
en cela à la conception chrétienne, c'est-à-dire rectilinéaire et téléologique de la temporalité, selon laquelle l'éternité paraît lorsque cesse le temps. Mais on décèle malgré tout chez Rahner des éléments ouvrant la voie à une conception positive de la temporalité humaine. Elle se calque sur un mouvement général de sa pensée qui consiste à passer, à l'occasion du traitement de quelques thèmes précis, d'une finitude négative à une finitude positive. Ce passage de la négativité à la positivité en regard de la finitude et du devenir est difficilement décelable dans l'œuvre de Rahner. Un thème, cependant, est extrêmement révélateur sur ce sujet, à savoir le problème de l' "individuel ». Chose très importante dans le cas d'une comparaison avec Nishida chez qui se retraçait exactement la même préoccupation, il faut éviter soigneusement de confondre l'«individuel» avec le «particulier»:

L'individu est singularité, c'est-à-dire justement pas individu particulier. Le particulier n'est tel que subsumé sous une loi générale, qui le détermine comme particulier. Le singulier est en revanche autodéterminé : il contient en lui la présence (transcendante) de l'universel. Le particulier est donc subsumé sous un concept générique, car le genre implique l'espèce; il est le fruit d'une division. Le singulier n'est pas subsumable sans être transformé en particulier. L'universel qu'il contient n'est pas un genre dont il serait l'espèce. (Piguet 1975, paragr. 3013)

Une science de l'individuel est impossible dans les cadres d'une théorie de la connaissance qui manie de semblable façon les catégories du général et du particulier. Elle «va se greffer sur les procédés de la connaissance au lieu de se greffer sur les propriétés de l'individuel dont il peut y avoir connaissance» (Piguet 1975, paragr. 1321). Dans ces conditions, l'individuel est considéré comme une simple entité réitérable. Rahner essaya de se dégager du rationalisme de la métaphysique scolastique et de comprendre l'individuel autrement que par le biais des idées générales, chose qui ne s'effectua pas sans un certain flottement terminologique qui témoigne de ses efforts en vue de passer de la négativité à la positivité. Il mentionne par exemple dans L'Homme à l'écoute du verbe qu'un processus libre tel que l'autocommunication de Dieu «n'est pas simplement le cas particulier (Fall) d'une loi générale (Algemeine) »; il est donc "l'opposé de l'objet des sciences de la nature, c'est-à-dire d'une connaissance qui cherche à élaborer des lois nécessaires et générales, expliquant totalement le particulier» (Rahner 1968c, 203). L'histoire elle-même existe seulement «là où l'unicité et la valeur personnelle l'emportent sur l'assujettissement aux lois générales et aux grands nombres, donc là où il y a liberté » (Rahner 1968c, 232). 
Rahner, critiquant par la même occasion les habitudes de pensée tant philosophiques que théologiques, revint à de nombreuses reprises sur cette question de l'irréductibilité de l'individuel aux lois générales et de l'impossibilité d'en faire un simple cas d'application de ce type de lois. Il constate par exemple que

quand il s'agit de réflexion philosophique et théologique et non de l'existence chrétienne pratique et concrète, l'individuum est chez nous conçu comme un «cas» particulier d'une essence générale. Le singulier, l'individuum, est considéré uniquement comme un cas d'application, d'espèce numérique (quantitative), d'une idée générale. Si ce cas particulier présente des singularités par rapport à l'idée générale, elles sont considérées comme indifférentes et sans importance et même le plus souvent, dans une métaphysique scolastique (sauf exceptions), comme une réalité simplement limitatrice et restrictive. [...] Je déclare fausse l'opinion selon laquelle, là même où il s'agit de l'esprit, de la conscience et de la liberté, l'individuum pourrait être conçu comme un simple cas particulier d'une règle universelle, comme un simple cas restrictif d'une essence générale, comme une réalité simplement numérique, comme un simple numéro dans une série. ( «L'exigence de Dieu et la personne ", Rahner 1970c, 49-50; voir aussi Rahner 1966b, 107, 143 et 155156)

La nécessité, prônée par Rahner, de la mise en vigueur d'une manière autre de comprendre l'individuel implique un passage radical, celui d'une métaphysique s'articulant dans le cadre logique du général et du particulier, à une attention à l'histoire qui vise l'individuel à partir de lui-même. C'est là la condition sine qua non d'une prise au sérieux du caractère historique de la révélation et, par conséquent aussi, de la mise à l'écart de tout type de gnose. Rahner soutient avec constance que

ce qui est annoncé dans le christianisme, ce n'est pas une vérité universelle, nécessaire et abstraite, qu'on pourrait atteindre indifféremment de n'importe quel point de l'histoire, parce qu'elle est fixée au ciel des idées, dans une éternelle indépendance et une lumière toujours égales; le christianisme est d'abord l'événement dans lequel Dieu traite avec nous dans sa grâce. C'est un événement qu'il est impossible de déduire de quoi que ce soit, mais qui doit être expérimenté dans sa réalité et sa liberté, et qui occupe de ce fait un point tout à fait déterminé et localisé dans l'histoire de l'homme. ( «Écriture et tradition. À propos du schéma conciliaire sur la révélation divine », Rahner 1967a, 80) 
Sur cette question de l'individuel qui excède radicalement le rapport logique du général et du particulier, et qui vise à mettre l'accent sur l'histoire et la liberté, la pensée de Rahner est remarquablement similaire à celle de Nishida. Au cours de la période marquée par le développement de la logique du lieu (1926-1930), Nishida tenta, à partir d'une prise au sérieux de l'individuel aristotélicien et d'une réinterprétation de l'universel concret hégélien, d'établir un type de «logique» qui excède les cadres de la simple articulation entre l'universel de type abstrait et le particulier, le second étant un simple cas d'application du premier (voir Tremblay 2000a, 16-24). Dans l'esprit de Nishida comme dans celui de Rahner, ce type de logique abstraite ne permet aucune connaissance de l'individuel qui, seul, est véritablement réel. La logique du lieu octroie d'entrée de jeu une positivité à l'individuel dont le caractère «irréitérable» (Rahner dirait «fini») est reconnu comme le plus important lorsqu'il est question de la liberté, de l'agir et de la personnalité humaines.

Étant donné sa tradition intellectuelle et spirituelle, ce n'est pas dans tous les domaines de la théologie que Rahner sut reconnaître à sa juste mesure toutes les implications de la valeur irremplaçable de l'individuel pour la pertinence d'une religion chrétienne qui, malgré sa tendance à grever négativement le fini, clame son aspect historique. Cependant, sa préoccupation à l'égard des deux dogmes les plus importants du christianisme que sont la révélation et l'incarnation l'obligèrent à reconsidérer l' 'insignifiance du pur contingent singulier» (Rahner 1985, 37) et à lui accorder une positivité sans laquelle les énoncés centraux de la foi n'auraient plus aucune pertinence. Bref, parler d' «historicité de la révélation» doit aller de pair avec une «positivation» de la finitude.

Outre la théologie dogmatique, il est un autre lieu où Rahner effectua le passage d'une finitude négative à une finitude positive; il s'agit de ses ouvrages traitant plus proprement de spiritualité. Dans Appels au Dieu du silence, la section 2 a exposé avec suffisamment d'évidence que, tout ce qui concerne l'être humain et son existence quotidienne est considéré d'un point de vue éminemment pessimiste et empreint d'une négativité qui ne manque pas d'étonner de la part d'un théologien qui se voulut le chantre d'une religion du salut. Or, et c'est là un fait d'un grand intérêt, ce même livre présente deux modes de pensée concomitants; d'un côté, cette conception négative de la finitude, et de l'autre ce que j'ai qualifié autrefois d'approfondissement de l'existence humaine au moyen d'une exacerbation des contraires (Tremblay 1992a, 252-285). Qu'est-ce à dire? 
Devant sa finitude et les souffrances qui sont son lot quotidien en ce «bas-monde ", l'être humain est le plus souvent tenté de fuir son existence afin d'essayer, malheureusement en vain, de rejoindre un dieu situé dans un au-delà du monde. Mais une autre attitude est possible, qui consiste en un approfondissement des limites et des souffrances de l'existence puisque c'est là, et nulle part ailleurs, qu'est possible un rapprochement avec Dieu qui, alors, cesse d'être considéré comme une entité absolument hors de portée de la sphère humaine. Ce niveau déboute le réflexe de fuite devant une existence dont la prise en compte et l'approfondissement permettent la découverte de Dieu là où l'on n'aurait pas songé à le chercher. Le mystère de Dieu s'ouvre depuis le fond de la douleur et sa vie jaillit de la mort ellemême. Lorsque l'être humain, dit ailleurs Rahner, "réalise une ouverture radicale de sa vie à Dieu et à une vie éternelle qu'il envisage comme sa propre espérance, du même coup son rapport aux réalités et aux tâches de ce monde se modifie notablement: se produisent alors une libération et un allègement, et s'ouvre l'accès à la clarté » (Rahner 1985, 87). Rahner revint à plusieurs reprises dans l'ensemble de son œuvre sur le fait que la prison étroite et limitée de l'existence humaine finie est le lieu où se laissent percevoir la lumière et l'immensité de Dieu (voir, par exemple, Rahner 1966e, 20 ; 1966f, 34). La rencontre avec Dieu ne s'effectue donc plus au moyen d'une élévation quasi spatiale vers le haut car il se situe toujours-déjà avec l'être humain et le monde.

Le thème privilégié qui, dans Appels au Dieu du silence, permet ce nouveau type de rapport avec le monde du divin est celui de l'amour. Faisant échec à l'impossible réconciliation d'une série d'oppositions de type dualiste, il permet l'ouverture à la vie même de Dieu au sein de l'existence quotidienne: "Lorsque nous aboutissons à Toi dans l'amour, dans le véritable amour, nous sortons en quelque manière de l'étroitesse de notre nature finie, et il nous arrive de trouver le bonheur dans notre vie quotidienne, au milieu même des restrictions de notre pauvre existence limitée» (Rahner 1966d, 24). Par «sortie de la nature finie », Rahner n'entend plus une fuite de l'existence mais bien plutôt son approfondissement dans ses dimensions de limite, de finitude et de pauvreté. Le thème de l'amour lui permet de dire, faisant une fois de plus référence à Augustin: "Toi qui, par ton amour, occupe le centre même de mon cœur, et qui est plus proche de moi que je ne le suis à moi-même» (Rahner 1966d, 16).

Du fait de sa finitude, tous les efforts déployés par l'être humain en vue de rejoindre Dieu sont voués à l'échec et son éloignement paraît insurmontable: «Moi-même, ai-je réussi à m'approcher de Toi dans ma propre 
vie, ou bien, tout effort en ce sens a-t-il simplement abouti à me faire ressentir plus violemment l'amertume dont Tu abreuves mon âme par ton éloignement? » (Rahner 1966d, 123). Il apparaît maintenant que c'est précisément l'éloignement de Dieu qui suscite l'amour, lequel à son tour est cela même qui permet le rapprochement avec Dieu: «Plus l'immensité de Ton Être s'éloigne de mon propre néant, et plus il stimule l'audace de mon amour» (Rahner 1966d, 17). Ce qui signifie que "proximité et distance par rapport à Dieu grandissent dans la même mesure (non dans une mesure inverse)» («Essai d'une esquisse d'une dogmatique», Rahner 1966a, 33, n. 1). L'accent mis par Rahner sur l'impossibilité d'une connaissance générale de l'individuel fut porté à son achèvement dans L'Homme à l'écoute du verbe (dont la rédaction est légèrement postérieure à Appels au Dieu du silence) lorsqu'il présenta l'amour comme la lumière de la connaissance du fini: «Une connaissance du fini devient aveugle si elle n'admet pas qu'elle peut se réaliser uniquement dans l'amour»(Rahner 1968c, 177).

Avec cela est pleinement accompli le passage d'une conception négative à une conception positive de la finitude. Si c'est du sein du monde et de l'existence humaine que surgit la lumière de Dieu, on n'est plus autorisé à les laisser en arrière en faveur d'un monde situé dans l'au-delà et de promesses de vie éternelle qui, plus souvent qu'autrement, justifient la paresse voire la lâcheté devant les tâches à accomplir dans le monde réel. "S'il existe, finit par reconnaitre Rahner, un chemin capable de me conduire jusqu'à Toi, ce chemin doit nécessairement traverser ma vie quotidienne » (Rahner 1966d, 76). D'où cette définition originale de la foi en Dieu: «Croire en Toi signifie simplement: suivre le chemin obscur et ténébreux de ma pauvre vie humaine, avec ses demeures mal éclairées, où vient s'infiltrer, cependant, la lumière de Ta vie éternelle» (Rahner 1966d, 89-90).

L'importance de la finitude de l'existence humaine intramondaine apparaît de manière éminente lorsqu'on considère qu'avec la figure de Jésus, Dieu lui-même est devenu un être humain fini:

Tu T'es emparé de ce que nous fuyons; Tu as commencé par accepter ce qui, nous semble-t-il, devait prendre fin avec Ta venue: notre vie fragile, limitée et vouée à la mort. Tu as pris sur Toi notre nature humaine, sans vouloir l'anéantir ou la transformer visiblement, au point de la faire apparaître transfigurée, déifiée aux yeux de tous [...]. Tu as accepté notre vie telle qu'elle est en réalité, et Tu l'as faite tienne. Tu l'as laissée s'écouler sur cette terre. Tu l'as saisie avec précaution, évitant qu'une seule goutte de son amertume ne se perde avant que Toi, Tu n'en aies bu le calice jusqu'à la lie. (Rahner 1966d, 125) 
Grâce à l'irruption de Dieu en ce monde qu'est l'incarnation, l'un et l'autre sont mis en rapport définitif. Dieu se donne à l'humain comme son avenir absolu, mais au stade où le monde historique est encore en devenir. Ainsi, l'incarnation du Verbe de Dieu en Jésus ne signifie rien d'autre que ceci: "Jésus-Christ réalise dans sa personne le don de lui-même que Dieu fait au monde pour être son avenir absolu» (Rahner 1966e, 158). Dieu est le terme du développement du monde et le garant de son dynamisme.

Or, et c'est là l'élément le plus important pour notre propos, ce devenir-humain de Dieu a directement à voir avec la temporalité. Rahner reconnaît que, dans le christianisme, le temps n'est plus celui de la pensée antique, c'est-à-dire un cycle monotone accumulant les saisons, les années, les siècles et les civilisations. Il n'est pas un devenir sans substance "où rien ne se passe parce que tout y passe ». Ce qui est arrivé avec Jésus a modifié fondamentalement la nature du temps, sans pour autant abolir sa structure et son déroulement phénoménal. Le temps s'est vu octroyer une signification transcendante dans la mesure où l'éternité a été introduite dans le présent lui-même.

Grâce à Jésus-Christ, le temps est devenu ce qu'il devait être, c'est-à-dire un temps racheté (Rahner parle en ce sens d'une «rédemption du temps»). L'humanité déchue n'avait connu jusqu'alors que «cette morne succession et cet écoulement inconsistant de fragments de durée dont chacun, pour exister, doit jeter dans la mort du passé celui qui le précède tout en disparaissant à son tour, car l'avenir est là qui le pousse, marqué d'ailleurs déjà lui-même du signe de la mort» (Rahner 1966f, 14). Mais le temps possède déjà "un centre de gravité capable de conserver le présent et de l'intégrer dans l'avenir, un centre qui irradie sur le présent la plénitude d'un avenir qui est une réalité déjà acquise, un centre qui assure au présent une permanence vivante et qui l'oriente irrésistiblement vers l'avenir éternel» (Rahner 1966f, 14³). Et cela grâce au Christ qui s'est inséré dans le temps prisonnier de lui-même, le Christ qui était hier, qui est aujourd'hui et qui sera dans l'éternité $(\mathrm{He} 13,8)$.

Dans cette perspective, la foi ne consiste pas dans un simple enregistrement par l'esprit d'un événement passé et de ses conditionnements supratemporels. Elle ne se ramène pas, non plus, à prendre connaissance d'un nouvel événement de l'histoire du salut qui adviendrait plus tard, dans un avenir dénué de toute réalité actuelle. Car «le salut lui-même [arrive au

3. Au sujet de la permanence du présent (dans l'éternité), voir p. 220-222. 
croyant] du lointain des âges, rejoint son présent, devient dans son histoire quelque chose d'actuel» (Rahner 1966f, 16). Grâce à la foi, le croyant devient contemporain de Jésus, il vit et meurt en lui. Autrement dit, le Christ est déjà présent dans le croyant, même s'il est son avenir. Grâce à cet avenir déjà arrivé en lui, le croyant commence à être ce qu'il sera le jour de son achèvement. L'événement ayant commencé avec l'incarnation de Jésus l'affecte maintenant et s'achèvera lors du retour du Christ. Si l'avenir du croyant est le Christ et si le Christ est en lui, alors il tient déjà son avenir. Rahner va jusqu'à considérer que, dans ces conditions, la foi est le pouvoir de bannir la crainte devant l'écoulement du temps.

Ce qui précède permet de conclure que Jésus-Christ réunit en sa personne Dieu et l'humain, l'absolu et le contingent, l'éternel et l'histoire. Avec sa venue, le passé de chaque être humain est replacé dans le cadre d'un avenir éternel et sans limite puisque Dieu comme avenir infini entre dans le cœur de l'humain. En ce sens, les chrétiens doivent à la fois s'engager dans le monde et être tendus vers un au-delà du monde et de l'histoire.

Avec l'incarnation a été inauguré un avenir dont le sens et le contenu sont fixés. Seule la manifestation de ce qui est déjà là de manière permanente représentera une nouveauté. Jésus, qui est " hier, aujourd'hui et dans les siècles » a inauguré la fin des temps. Grâce à lui, le temps est déjà arrêté: "Nous sommes arrivés à la fin des temps» (1 Co 10,11; voir Rahner 1966d, 128). Le temps qui demeure dans le monde est celui de l'Avent de Dieu. Au dernier jour, il disparaîtra pour laisser place à Dieu et à son éternité. Cela signifie que l'humain vit dans les derniers temps, ceux de la plénitude des temps. Il est vrai que de par sa finitude, il est rivé au temps par le poids de lourdes chaînes; mais il attend avec joie le monde à venir, c'est-àdire la venue définitive de Jésus glorifié. En ce sens, le christianisme est une religion de l'avenir, une religion de l'humain nouveau et éternel.

Terminons l'exposition de ce passage effectué par Rahner d'une temporalité négative à une temporalité positive en faisant intervenir la notion nishidienne de maintenant éternel qui constitue l'un des points de contact intéressants entre les deux auteurs. Dieu, dit Rahner, est plus puissant que le temps, "cette créature qu'il a fait surgir du néant pour la racheter et l'intégrer à son éternité» (Rahner 1966f, 20). Il semble être un «éternel aujourd'hui ", distant de tout et renfermant en lui tous les temps et tous les changements. Il continue d'être ce qu'il était hier et a déjà dépassé l'avenir le plus lointain de l'humain. L'éternité est un "présent unique », un " "maintenant" toujours identique et toujours nouveau qui, situé dans la sphère du supratemporel, ne voit que de très haut et de très loin le déroulement du 
temps» (Rahner 1966d, 228). Mais avec l'incarnation, le monde éternel, l'éternel «aujourd'hui» se rapproche "du lointain abîme de nos jours mortels ", «des sombres vallées de notre vie éphémère " (Rahner 1966d, 228). Dès lors, fait remarquer Rahner au croyant, «le "maintenant" éternel est en toi, ce "maintenant" que rien ne limite plus désormais, ni en avant ni en arrière, et qui a déjà commencé à rassembler en lui la gerbe de tous tes instants terrestres» (Rahner 1966d, 20). Avec cette notion de maintenant éternel, l'insistance est mise non plus uniquement sur un avenir absolu situé dans un au-delà de l'histoire et de la temporalité, mais sur la venue de Dieu, éternel aujourd'hui, dans l'heure présente. Si l'on prend au sérieux le devenir-humain de Dieu, la temporalité humaine et l'éternité ne peuvent que se retrouver ainsi conjointes. Autrement, Dieu serait un Dieu uniquement transcendant et intemporel, sans rapport avec l'existence humaine.

L'important, dans ce contexte-ci, est que le rapport de Dieu à l'histoire ne doit pas être conçu comme un événement s'étant déroulé dans un passé lointain (le Jésus historique) et qui serait désormais sans rapport avec l'humain, si ce n'est par le biais de la remémoration. Jésus continue d'accompagner l'histoire humaine au fil, précisément, de la temporalité. Ce rapport ne doit pas non plus être limité au retour de Jésus dans un avenir transterrestre (la parousie). Dans un cas comme dans l'autre, c'est-à-dire qu'on conçoive la venue de Dieu comme un événement passé ou comme un événement encore à venir, on évacue complètement le moment présent (au sens nishidien) de la temporalité humaine, le seul qui soit véritablement réel. Par "présent» est entendu ici non pas le chronos du temps rectilinéaire, mais ce que la théologie appelle le kairôs et qui, à ce niveau, présente des ressemblances marquées avec le présent absolu nishidien.

Dire que la rencontre avec Dieu s'effectue dans le moment présent enlève à ce dernier son caractère évanescent et contingent. Lorsqu'on conçoit d'une part l'incarnation dans un passé révolu, et d'autre part l'avenir absolu dans une atemporalité transterrestre, l'écoulement du temps ne peut qu'être marqué par la caducité et la contingence; il ne peut qu'être évalué avec un pessimisme dont Rahner est loin d'avoir la paternité dans le christianisme. Mais si l'on adopte la position d'une venue de Dieu conjointe à l'histoire et à la temporalité humaine - perspective qui, il va sans dire, ne se réduit pas à faire de Dieu lui-même un élément fini et contingent de cette histoire -, il faut redonner à la temporalité, notamment au présent, toute sa prégnance. En effet, où et quand l'humain peut-il vraiment rencontrer Dieu, si ce n'est dans son histoire humaine, et plus particulièrement dans le moment présent? La simple remémoration d'une série 
d'événements s'étant déroulés dans le passé (fussent-ils aussi importants que l'autocommunication de Dieu) et sans rapport avec l'expérience humaine présente ne concerne plus l'humain, sauf sous le mode d'une série de vérités à croire. D'autre part, la promesse d'une vie éternelle et d'un retour du Christ advenant depuis l'extrémité de l'histoire et de la temporalité, c'est-à-dire à leur terme, ne peut qu'entraîner une vision extrêmement pessimiste de la vie et du monde "d'ici-bas ", désormais considérés uniquement sous leurs aspects transitoires. Bref, ni le passé, ni l'avenir, pris soit séparément, soit ensemble, ne peuvent être les lieux d'une véritable rencontre avec Dieu. Sans la prise en compte du présent comme lieu précis de la venue de Dieu, l'humanité et les tâches terrestres d'avenir n'ont plus qu'une importance secondaire.

\section{Pour une eschatologie topologique}

Le type de critique de la religion qui vient d'être fait n'est pas nouveau. Ce qui l'est, c'est l'inspiration de la philosophie de Nishida. En comparant Rahner et Nishida, le but poursuivi n'était pas d'inféoder l'un à l'autre mais d'apporter à la théologie de Rahner, et par extension à la théologie chrétienne, un éclairage nouveau. Cependant, cet éclairage n'était pas insolite au point de rendre la pensée de Rahner méconnaissable ou de la biaiser dans un sens qui lui est étranger. Car comme le montrent certains des passages qui ont été évoqués, Rahner eut lui-même de fortes intuitions dans le sens d'une venue de Dieu au cœur même du présent qui allèrent de pair avec l'importance qu'il s'efforça en fin de compte de placer sur la finitude et la temporalité humaines.

Il est évident que les paradoxes de la théologie de Rahner, la simultanéité qu'on y trouve d'une tendance à fuir le monde et d'une insistance sur l'histoire et la matière, ne peuvent être résolus que dans le type de christologie qu'il mit en œuvre. C'est là qu'il réussit dans une large mesure à faire correspondre tous les thèmes considérés contradictoires du point de vue d'une finitude et d'une temporalité négatives. Pourquoi, alors, ne développa-t-il pas jusque dans leurs ultimes conséquences toutes les potentialités impliquées dans le concept — qu'il forgea lui-même — d'un devenir-humain de Dieu? Certes, il montra que le Dieu infini est devenu fini en se niant lui-même, qu'il s'est incarné précisément «dans » l'histoire humaine et «dans » un être humain fini. Là se trouve le caractère le plus original de sa théologie. Mais il n'étendit pas à d'autres thèmes les possibilités ouvertes par sa christologie. Peut-être n'était-il tout simplement pas en possession de 
l'appareil conceptuel qui lui eût permis de le faire. Il eut pourtant des intuitions justes. Mais enserré qu'il était dans son mode de pensée, il ne put aller au bout de leurs implications. Si l'autocommunication de Dieu a rendu l'humain apte à rencontrer Dieu dans l'histoire et au sein de sa propre temporalité, pourquoi ne pas avoir levé totalement le caractère négatif qui les alourdissait encore et ne pas avoir fait définitivement échec au réflexe de fuite qui s'épuise à essayer de rejoindre un Dieu situé au-delà de la temporalité et de l'histoire humaines, bien que cela soit à l'évidence peine perdue ? Il est vrai que Rahner insista sur le fait que Dieu se donne dès maintenant à l'humain et que l'avenir rejoint le présent. Nonobstant, on ne peut s'empêcher de demeurer sous l'impression que ce procédé vise encore à tirer le présent hors de lui-même, vers l'avant, et à arracher l'humain à sa temporalité. Là se trouve précisément le problème structurel de la théologie de Rahner et, de manière plus générale, de l'ensemble de l'eschatologie chrétienne.

Il est cependant possible, en s'inspirant de la conceptualité nishidienne, d'élargir les champs d'application possibles du concept de devenir-humain. Cela a déjà été fait dans une large mesure dans la section précédente mais il importe de revenir ici sur quelques thèmes précis qui fourniront par la même occasion matière à conclusion. Mon intention est d'essayer de montrer que la logique du basho et la conception nishidienne de la temporalité permettent de repenser avec profit les conditions a priori - établies par Rahner au moyen de sa méthode et de son anthropologie transcendantales - de possibilité dans l'esprit humain de l'accueil d'une révélation possible de Dieu. Cette méthode a l'avantage de ne pas s'embarrasser des disputes théologiques pour s'intéresser d'abord aux structures de l'existence et de l'esprit humains.

Je dirai d'abord qu'à l'évidence, la conception négative du monde et de l'humain qui a été remise en question précédemment était étrangère à Nishida. Il faut cependant hésiter à aller jusqu'à parler dans son cas d'une conception "positive ", puisque le positif est le simple opposé du négatif et se situe par conséquent dans le même cadre de pensée. Nishida s'attacha tout simplement à analyser l'existence telle qu'elle est, en dehors de tout dualisme métaphysique opposant le fini à l'infini, la matière et le corps à l'esprit, l'humain à Dieu, et le temps à l'éternité. Il articula conjointement toutes ces réalités dans le «lieu du néant » et montra qu'elles entretiennent des rapports de «détermination» et de réciprocité. 
Sur la base de cette "anthropologie» nishidienne, la doctrine chrétienne de l'incarnation peut être révisée non pas comme une tentative vouée d'entrée de jeu à l'échec - de réconciliation de termes entretenant les uns par rapport aux autres des relations dualistes, mais bien plutôt comme un événement relationnel occasionnant des déterminations ou modifications significatives tant chez l'humain que chez Dieu. En ce sens, le «devenirhumain de Dieu » peut être conçu comme une autodétermination de Dieu, par laquelle il se modifie de manière telle qu'il peut entrer en communication radicale avec l'être humain, jusqu'à devenir lui-même humain. Par "autodétermination", il ne faut pas entendre une représentation spatiale selon laquelle Dieu demeurerait un point fixe duquel émanerait, en une ligne directe, quelque chose. Bien plutôt, c'est Dieu lui-même qui, en s'autodéterminant, devient humain. Ce que la théologie appelle la "grâce » signifie dans ce contexte qu'en faveur de cette "autodétermination », l'humain est désormais lui aussi radicalement transformé. Cette interprétation, qu'il reste à développer dans toutes ses ramifications possibles, permet de faire voir ici les importantes potentialités déjà en germe chez Rahner, mais que son cadre de pensée ne lui permit malheureusement pas d'explorer à fond.

Autre exemple de réinterprétation de Rahner à partir de Nishida: il est possible d'établir un parallèle entre la doctrine de la création continuée d'une part, et le temps comme autodétermination du maintenant éternel d'autre part, en faisant toutefois très attention à ceci: ce que Nishida appelle le «maintenant éternel » n'est pas l'équivalent de Dieu, mais bien du néant absolu; en ce sens, il est intrinsèquement relié à la notion d'autonégation. Cette précaution prise, ce parallèle contribue au renversement d'une éternité située dans le domaine supratemporel, sans pour autant lui accorder un statut intratemporel. Encore une fois, l'un des problèmes qui sont ici en jeu en est un de représentation et de conception du monde qui peut être résolu avec une insistance sur les notions nishidiennes d'autodétermination et de détermination réciproque. En effet, si Rahner se montra incapable d'accorder à la temporalité humaine en tant que telle un statut définitivement positif et s'il ne put que lui concéder après coup une certaine positivité grâce à l'intervention divine, c'est certainement en raison de son type de représentation de l'éternité.

Ce même problème de représentation est-il totalement absent de la notion nishidienne de maintenant éternel comme autre nom du néant absolu ? Nishida parvint-il vraiment à dépasser tout type de représentation spatiale dans son traitement de la temporalité ? En un certain sens non, puisqu'il compare le présent absolu et le néant absolu à un cercle infiniment 
grand dont le centre est partout, la circonférence nulle part. Mais la comparaison atteint rapidement sa limite car ce cercle étant infiniment grand, il est précisément impossible d'en concevoir les limites, et donc de le représenter. La notion de cercle infiniment grand est pour ainsi dire un cas limite de représentation en ce sens qu'aussitôt posée, elle défie tout type de représentation. Ne restent que les relations entre d'une part le présent absolu, le maintenant éternel et le néant absolu, et d'autre part ce qui se situe en eux, à savoir le temps, les mondes physique, biologique et historique, de même que l'être humain.

\section{Références}

NisHIDA, K. (1978),『西田幾多郎全集』(NKZ; Nishida Kitarō zenshū, CEuvres complètes de Nishida Kitarō), 19 volumes, Tokyo, Iwanami Shoten. (2003), L'éveil à soi, Paris, CNRS Éditions, trad. par J. Tremblay.

Piguet, J.-C. (1975), La Connaissance de l'individuel et la logique du réalisme, Neuchâtel, La Baconnière.

RAHNER, K. (1959), Écrits théologiques, tome 1, Paris, Desclée de Brouwer. (1960), Écrits théologiques, tome 2, Paris, Desclée de Brouwer. (1963), Écrits théologiques, tome 3, Paris, Desclée de Brouwer. (1965), Mission et grâce, tome 3, Paris, Mame.

- (1966a), Écrits théologiques, tome 4, Paris, Desclée de Brouwer. (1966b), Écrits théologiques, tome 5, Paris, Desclée de Brouwer. - (1966c), Écrits théologiques, tome 6, Paris, Desclée de Brouwer. Salvator. (1966d), Appels au Dieu du silence. Dix méditations, Mulhouse, (1966e), Est-il possible aujourd'hui de croire? Dialogue avec les hommes de notre temps, Paris, Mame.

- (1966f), L’homme au miroir de l'année chrétienne, Paris, Mame. - (1967a), Écrits théologiques, tome 7, Paris, Desclée de Brouwer. (1967b), Écrits théologiques, tome 8, Paris, Desclée de Brouwer. (1968a), Écrits théologiques, tome 9, Paris, Desclée de Brouwer. (1968b), L’Esprit dans le monde. La Métaphysique de la connaissance finie chez Thomas d'Aquin, Paris, Mame. 
(1968c), L'homme à l'écoute du verbe. Fondements d'une philosophie de la religion, Paris, Mame.

(1970a), Écrits théologiques, tome 10, Paris, Desclée de Brouwer. (1970b), Écrits théologiques, tome 11, Paris, Desclée de Brouwer/ Mame. Mame.

(1970c), Écrits théologiques, tome 12, Paris, Desclée de Brouwer/ (1983), Traité fondamental de la foi. Introduction au concept du christianisme, Paris, Centurion.

(1985), Aimer Jésus, Paris, Desclée.

RAHNER, K. «Pour une théologie de l'enfance », L'Anneau d'or, 110, p. 428439.

Rahner, K et H. Vorgrimler (1970), Petit dictionnaire de théologie catholique, trad. par P. Démann et M. Vidal, Paris, Seuil.

Tremblay, J. (1989), «La problématique transcendantale. D’une vision ptolémaïque à une vision copernicienne du monde ", Approches, 12, p. 3947.

(1990), «La question de l'individuel chez Karl Rahner », Approches, 17, p. 29-36.

(1992a), Finitude et devenir. Fondements philosophiques du concept de révélation chez Karl Rahner, Montréal, Fides (Héritable et projet; 47).

(1992b), «Le dialogue interreligieux. Quelles logiques? ", Nouvean Dialogue, 91, p. 22-23.

(1997), «La transcendance immanente chez Nishida Kitarō et Karl Rahner », Sciences Religieuses, 26/2, p. 1-12.

(2000a), Nishida Kitarō. Le Jeu de l'individuel et de l'universel, Paris, CNRS Éditions.

- (2000b), La Relation et son lieu. Introduction à la philosophie de la relation de Nishida, Beauport, MNH/Anthropos.

- (2007a), Introduction à la philosophie de Nishida, Paris, L'Harmattan.

(2007b), Auto-éveil et temporalité. Les Défis posés par la philosophie de Nishida, Paris, L'Harmattan.

(2007c), L'Être-soi et l'être-ensemble. L'auto-éveil comme méthode philosophique chez Nishida, Paris, L'Harmattan. 
Tremblay, J., dir. (2008), Philosophie japonaise $d u X X^{\mathrm{e}}$ siècle, Laval théologique et philosophique, 64/2, p. 233-573.

(2010), Enjeux de la philosophie japonaise du XX $x^{\mathrm{e}}$ siècle, Montréal, Presses de l'Université de Montréal.

\section{Résumé}

De nombreux points de comparaison sont possibles entre la philosophie de Nishida Kitarō (1870-1945) et la philosophie de la religion de Karl Rahner (1904-1984), notamment concernant la temporalité, thème à propos duquel ces deux auteurs réfléchirent radicalement. En réexaminant la notion rahnérienne d'avenir absolu à la lumière de la notion nishidienne de présent absolu, il est possible d'apporter une solution au problème suivant: rejoint-on Dieu (Rahner) ou l'absolu (Nishida) dans un avenir situé au-delà de la temporalité ou bien dans l'instant présent, compris comme le lieu même du jaillissement de la temporalité? Dans la mesure où elle s'appuie sur une philosophie du néant absolu, la pensée de Nishida permet de dégager la philosophie de la religion de Rahner des cloisons de l'onto-théo-téléologie qui l'enserrent et, la plaçant ainsi sur un horizon plus vaste, la rend apte à exprimer, d'une manière qu'on espère nouvelle, les nombreuses potentialités qu'elle recèle encore. En retour, une lecture de la temporalité nishidienne à travers le regard de Rahner permet de l'approfondir et de faire ressortir des éléments inédits.

\section{Abstract}

Many points of comparison are possible between the philosophy of Nishida Kitaro (1870-1945) and the philosophy of Religion of Karl Rabner (19041984), particularly temporality, a theme around which these two authors thought radically. In reconsidering Rahner's concept of absolute future in the light of Nishida's concept of absolute present, it is possible to solve the following problem: do we join God (Rabner) or the absolute (Nishida) in a future located beyond temporality or in the present instant, understood as the very place of emergence of temporality? Insofar as it is based on a philosophy of absolute nothingness, Nishida's philosophy allows to overtake the onto-theo-teleological character of Rahner's philosophy of religion and, situating it on a wider horizon, to make it fit to express, in a new way, its many hidden possibilities. In return, a reading of Nishida's temporality through the eyes of Rahner allows to deepen it and to bring out new elements. 